\title{
Systematic Review of Randomized Controlled Trials of Exercise Interventions Using Digital Activity Trackers in Patients With Cancer
}

\author{
Kerry Schaffer, MDa; Narmadha Panneerselvam, MD ; ; Kah Poh Loh, BMedSci, MBBCH, BAO; \\ lan R. Kleckner, PhDa; Richard Francis Dunne, MDa; Po-Ju Lin, PhD, MPH, RDa; Charles E. Heckler, PhDa; \\ Nicholas Gerbinoa; Lauren B. Bruckner, MD, PhDa; Eugene Storozynsky, MD, PhDa; Bonnie Ky, MD, MSCEd; \\ Andrea Baran, MS'; Supriya Gupta Mohile, MD, MSa; Karen Michelle Mustian, PhD, MPHa; and Chunkit Fung, MDa
}

\begin{abstract}
Background: Exercise can ameliorate cancer- and treatment-related toxicities, but poor adherence to exercise regimens is a barrier. Exercise interventions using digital activity trackers (E-DATs) may improve exercise adherence, but data are limited for patients with cancer. We conducted a systematic review examining the feasibility of E-DATs in cancer survivors and effects on activity level, body composition, objective fitness outcomes, health-related quality of life (HRQoL), self-reported symptoms, and biomarkers. Methods: We identified randomized controlled trials (RCTs) of E-DATs in adult cancer survivors published in English between January 1, 2008, and July 27,2017 . Two authors independently reviewed article titles $(n=160)$, removed duplicates $(n=50)$, and reviewed the remaining 110 articles for eligibility. Results: A total of 12 RCTs met eligibility criteria, including 1,450 patients (mean age, 50-70 years) with the following cancers: breast $(n=5)$, colon or breast $(n=2)$, prostate $(n=1)$, acute leukemia $(n=1)$, or others $(n=3)$. Duration of E-DATs ranged from 4 to 24 weeks, and the follow-up period ranged from 4 to 52 weeks, with retention rates of $54 \%$ to $95 \%$. The technology component of E-DATs included pedometers $(n=8)$; pedometers with smartphone application $(n=1)$, Wii Fit $(n=1)$, heart rate monitor $(n=1)$; and a wireless sensor with accelerometer, gyroscope, and magnetometer $(n=1)$. Adherence by at least one measure to E-DATs was $>70 \%$ in 8 of 8 RCTs. Compared with controls, E-DATs significantly improved patients' step count in 3 of 5 RCTs, activity level in 6 of 9 RCTs, and HRQoL in 7 of 9 RCTs (all $P \leq .05$ ), with no significant changes in biomarkers (eg, interleukin 6, tumor necrosis factor $\alpha, C$-reactive protein, c-peptide, lipid panel) in 3 RCTs. Duration of E-DAT was not significantly correlated with adherence or study retention. Conclusions: This systematic review shows that E-DATs are feasible to implement in cancer survivors. Future research should examine the optimal type, dose, and schedule of E-DATs for cancer survivors.
\end{abstract}

J Natl Compr Canc Netw 2019;17(1):57-63 doi: 10.6004/jnccn.2018.7082

'Wilmot Cancer Institute, University of Rochester Medical Center, Rochester, New York; ${ }^{b}$ Monmouth Medical Center, Monmouth, New Jersey; ${ }^{c}$ Cornell University, Ithaca, New York; and 'Hospital of the University of Pennsylvania, Philadelphia, Pennsylvania.
The number of cancer survivors, defined as individuals with cancer from diagnosis through death, ${ }^{1}$ has increased markedly in recent decades and will reach an estimated 18 million worldwide by $2022 .{ }^{2}$ Cancer survivors experience a wide range of physical and psychological symptoms due to their underlying malignancies and associated treatments. ${ }^{1,3,4}$ Exercise is a promising intervention to prevent and ameliorate cancer- and treatment-related toxicities. ${ }^{5}$ Prior studies, ${ }^{6-11}$ including a systematic review of 34 exercise interventions for patients with cancer during and after treatments, ${ }^{6}$ report that exercise in survivors improves strength, endurance, and cardiopulmonary function, thereby resulting in improved physical and mental health and health-related quality of life (HRQoL). Based on these benefits, efforts to integrate exercise interventions in cancer care are increasing. ${ }^{1,12}$ These interventions can be delivered in prescriptions that define exercise mode, duration, frequency, and intensity as part of routine cancer care.

Poor adherence to exercise interventions is a major barrier to achieving the optimal benefits of exercise. ${ }^{13-16}$ The presence of symptoms related to cancer and its treatments, including fatigue, depression, insomnia, and pain, may further hinder cancer survivors' exercise intervention adherence..$^{13}$ Multiple studies suggest that self-efficacy is an independent predictor of exercise adherence in the general population ${ }^{17,18}$ and in patients with illnesses, including chronic obstructive pulmonary disease, ${ }^{19}$ obesity, ${ }^{20}$ congestive heart failure, ${ }^{21}$ stroke, ${ }^{22}$ type 2 diabetes mellitus, ${ }^{23}$ and cancer. ${ }^{24}$ There is, therefore, interest in identifying ways of increasing self-efficacy.

Exercise interventions using digital activity trackers (E-DATs), including digital healthcare-related wearable sensors, digital processing applications (apps), or communication equipment, ${ }^{25}$ may improve self-efficacy of cancer survivors, leading to higher adherence to exer-

See JNCCN.org for supplemental online content. 
cise interventions. ${ }^{26}$ E-DATs can facilitate monitoring of exercise progress for cancer survivors in real time, providing them with virtual feedback to enhance motivation and address potential exercise barriers. ${ }^{26-28}$ Additional benefits of E-DATs include objective data collection, patient-tailored (or precision) interventions, and in some cases, ability to communicate virtually with healthcare providers regarding symptoms, exercise experience, or questions.

Use of E-DAT behavioral interventions is a rapidly expanding area of research and practice ${ }^{27-29}$ but has only recently been applied to cancer survivors. ${ }^{30,31}$ To improve understanding of this emerging field, we conducted a systematic review to summarize findings from randomized controlled trials (RCTs) of E-DATs in cancer survivors. The primary aim of this review was to evaluate retention and adherence rates to E-DATs. The secondary aim was to assess the effects of E-DATs on activity level, body composition, objective fitness outcomes, HRQoL, self-reported symptoms, and biomarker data.

\section{Methods}

\section{Search Strategy}

We conducted a search of PubMed, Embase, and the Cochrane Library to identify RCTs published in English between January 1, 2008, and July 27, 2017, that evaluated effects of E-DATs on patients with cancer (supplemental eAppendix 1 outlines search methodology; available with this article at JNCCN.org). The search date of January 1 , 2008, was selected to reflect the year that the first DAT technology (ie, Fitbit) became available to the public. ${ }^{32}$ This study was exempt from Institutional Review Board approval at the University of Rochester Medical Center.

\section{Inclusion and Exclusion Criteria}

Two authors (K.S., R.H.) reviewed the titles of publications found by the search $(\mathrm{n}=160)$, removed duplicated studies $(n=50)$, and then separately reviewed the remaining publications $(n=110)$ for studies adhering to the following inclusion criteria: (1) RCTs; (2) study populations of adults with cancer aged $\geq 18$ years or survivors of adult-onset cancer; (3) E-DATs (pedometers and other tracking devices); (4) interventions promoting exercise, defined as intentional, structured, and repetitive, and activity with a purpose to address physical fitness, including muscle endurance, strength, or balance; and (5) inclusion of at least one of the following outcomes: activity level (eg, step count or proportion of time spent at various levels of activity, including both "exercise," defined above, and "physical activity," defined as all energy expenditure through skeletal muscles ${ }^{33}$ ), body composition (eg, weight, body mass index [BMI], or lean body mass), objective fitness outcomes ( $\mathrm{eg}, \mathrm{Vo}_{2 \max }$, chest press repetitions, 2-minute walk distance), HRQoL, self-reported symptoms (eg, fatigue, sleep quality), or biomarker data. Studies were excluded based on the following criteria: (1) interventions did not use E-DATs, such as only relaxation (vs exercise) interventions, or exercise interventions that used technology solely for data collection (eg, sealed pedometer recording steps for baseline and follow-up) rather than as a component of the exercise intervention; (2) study samples consisted of $>50 \%$ caretakers or support persons; and (3) patients had benign or precancerous conditions. The literature search yielded 12 RCTs, with 14 publications ( 2 RCTs had 2 publications each) that met the inclusion criteria (Figure 1).

\section{Data Collection and Analyses}

Two authors (K.S., N.P.) independently extracted the following information and 2 authors (K.P.L., R.H.) confirmed the data: study population characteristics (eg, mean age, cancer type and stage, and cancer treatment modality), countries where the RCTs were conducted, type and dose (frequency, amount, and intensity) of exercise interventions, and duration, retention, and adherence rates to exercise interventions. Any adherence data (eg, attendance, intensity, step count) available were recorded. The following outcomes were collected: activity level, body composition findings, objective fitness outcomes, HRQoL, self-reported symptoms, and biomarker data.

Due to heterogeneity of outcomes across studies, we were unable to perform a meta-analysis. To assess the

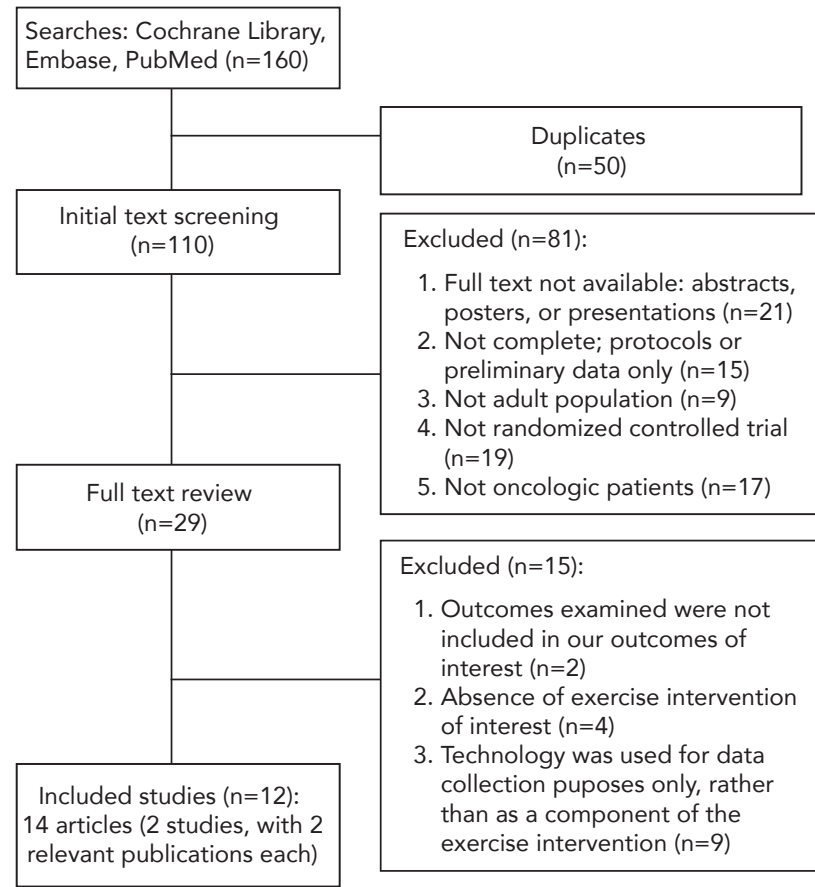

Figure 1. Study flow diagram. 
relationship between duration of E-DATs with retention and adherence rates, we used pairwise Spearman rank correlations.

\section{Results}

\section{Study Characteristics}

A total of 12 RCTs $^{34-47}$ met the eligibility criteria (supplemental eTable 1). The studies were conducted in the United States $(n=3)$, Australia $(n=2)$, Canada $(n=2)$, South Korea $(n=2)$, Denmark $(n=1)$, Sweden $(n=1)$, and the United Kingdom $(n=1)$. Five RCTs evaluated patients with breast cancer: 3 for stage I-III disease after or during adjuvant treatments, ${ }^{34,35,38} 1$ for stage I-III disease during neoadjuvant or adjuvant chemotherapy, ${ }^{43}$ and 1 for stage I-IV disease after adjuvant treatments. ${ }^{47}$ Two RCTs evaluated survivors of breast and colorectal cancers: 1 for stage I-IV disease during chemotherapy ${ }^{39}$ and the other for stage IIII disease after adjuvant treatments. ${ }^{42}$ The remaining 5 RCTs evaluated survivors of prostate cancer on androgen deprivation therapy $(\mathrm{n}=1),{ }^{45}$ patients with acute leukemia who experienced complete remission after induction chemotherapy $(\mathrm{n}=1),{ }^{44}$ and patients with various cancer types $(\mathrm{n}=3)^{40,41,46}$ One RCT included caretakers of survivors of various cancer types, with $<50 \%$ of the study population consisting of caregivers. ${ }^{41}$ Overall, 1,450 subjects $(1,417$ cancer survivors and 33 caregivers) were included in the 12 RCTs, with mean age ranging from 50 to 70 years.

\section{Characteristics of E-DATs}

Of the 12 RCTs, 6 investigated E-DATs with an in-person exercise training component, including supervised walking groups $(\mathrm{n}=1)$, training sessions at exercise facilities $(n=2)$, educational sessions about E-DATs $(n=2)$, and educational sessions about E-DATs with dietary counseling $(\mathrm{n}=1)$. The remaining 6 RCTs had a self-directed training component, including exercise recommendations conveyed via phone with written materials/protocol $(n=2)$, written printed materials $(n=1)$, smartphone app $(n=1)$, and DVD multimedia tool $(\mathrm{n}=2)$.

The technology component of E-DATs included pedometers only $(\mathrm{n}=8)$; pedometers combined with a smartphone app (SmartAfter-Care; $n=1$ ), Wii Fit (Nintendo; $n=1$ ), or heart rate monitor $(\mathrm{n}=1)$; and a wireless sensor with triaxial accelerometer, gyroscope, and magnetometer $(n=1)$ (supplemental eTable 1). The pedometers used included Silva EX Connect, ${ }^{39}$ Yamax Digi-Walker SW-200, ${ }^{34,35,43}$ Shinwoo DMC03, ${ }^{42}$ Omron Walking style Pro, ${ }^{44}$ InBody BAND ${ }^{47}$ or were not specified. ${ }^{36,40,45}$ Smart After-Care is a smartphone app (BIT Computer Co., Ltd., Seoul, Korea) used in conjunction with pedometers to monitor exercise level and provide feedback and education to cancer survivors about benefits of exercise. ${ }^{47}$ Wii Fit is an interactive, homebased, computerized exercise program launched by Nin- tendo in $2008^{48}$ that includes strength training, aerobics, and balance activities, and delivers the exercise program using various exercise modules tailored to each subject with increasing intensity over time. The wireless sensor with accelerometer, gyroscope, and magnetometer is an interactive motor adaptation balance training program. ${ }^{46}$ This technology-based system integrates data from wearable sensors into a human-computer interface designed for game-based motor adaptation training, providing real-time feedback on lower extremity motor functions during exercise. In most RCTs reviewed, the pedometer served as a patient-facing tool (vs physician-facing or patient-physician), ${ }^{49}$ allowing patients real-time, self-tracking feedback on their activity level. Control arms are described in supplemental eTable 1.

\section{Study Retention and Adherence to E-DATs}

After a follow-up ranging from 4 to 52 weeks for the 12 RCTs in this systematic review, the study retention was $54 \%$ to $95 \%$. All 8 RCTs with adherence data reported adherence of $>70 \%$ in at least one adherence measure, with adherence to E-DATs ranging from $33 \%$ to $96 \%$ (supplemental eTable 1). Median adherence to E-DATs was not significantly higher when it involved an in-person training component (75\%) compared with those involving solely self-directed training $(60 \% ; P=.40)$. No statistically significant correlations were found between the duration of E-DATs and adherence $\left(\mathrm{r}_{\text {Spearman }}=0.16 ; P=.75\right)$ or study retention $\left(\mathrm{r}_{\text {Spearman }}=-0.42 ; P=.17\right.$ ). Few trials provided data about dose of exercise level achieved compared with what was prescribed.

\section{Activity Level Outcomes}

Supplemental eTable 2 describes the activity level outcomes of the reviewed RCTs. ${ }^{34-37,39-45,47}$ Compared with control groups, E-DATs had significantly higher step counts in 3 of 5 RCTs and had significantly higher overall activity levels in 6 of 9 RCTs. Among 4 RCTs that evaluated both step count and overall activity level, E-DATs significantly improved both outcome measures in 2 of the 4 RCTs compared with control groups.

\section{Body Composition Findings and Objective Fitness Outcomes}

Supplemental eTable 3 describes the body composition findings other objective fitness outcomes of the reviewed RCTs. ${ }^{36-41,44-47}$ Of the 6 RCTs, ${ }^{36-39,41,45,47} 2$ reported significant improvement in body composition measurements in patients using E-DATs compared with controls: reduction in $\mathrm{BMI}^{41}$ and reduction in arm circumference at some, but not all, anatomic sites. ${ }^{38}$ Of 6 RCTs that evaluated fitness performance on specific physical tasks, $^{38,40,44-47} 3$ showed that E-DATs significantly improved fitness and physical performance compared with 
controls in the following measures: Short Physical Performance Battery, ${ }^{45}$ balance measures, ${ }^{46} 6$-minute walk test, ${ }^{44} \mathrm{Vo}_{2 \max }{ }^{44}$ sit to stand repetitions, ${ }^{44}$ and bicep curl repetitions. $^{44}$

\section{HRQoL and Self-Reported Symptom Outcomes} Supplemental eTable 4 describes the HRQoL and self-reported symptom outcomes of the reviewed RCTs. $^{34,35,38-40,42-44,46,47}$ Compared with control groups, E-DATs significantly improved HRQoL measures or self-reported symptoms (eg, fatigue, fear of falling, sleep quality) in 7 of the 9 RCTs measuring these outcomes.

\section{Biomarker Data}

Of the 12 RCTs, 3 examined effects of E-DATs on biomarker data outcomes compared with control groups..$^{37,39,44}$ None reported statistically significant differences between E-DAT and control for inflammatory markers, including interleukin 6 (IL-6), tumor necrosis factor $\alpha$ (TNF- $\alpha$ ), C-reactive protein (CRP), c-peptide, and cholesterol profile (fasting low-density lipoprotein, triglyceride, high-density lipoprotein, and total cholesterol).

\section{Discussion}

To our knowledge, this is the first systematic review of RCTs to evaluate feasibility of E-DATs and their effects on activity level, body composition, objective fitness outcomes, HRQoL, self-reported symptoms, and biomarker data in cancer survivors. We report that E-DATs are feasible to implement in cancer survivors, with $>70 \%$ adherence in at least one measure in 8 RCTs and a $54 \%$ to $95 \%$ study retention rate in 4 to 52 weeks of follow-up. Compared with controls, E-DATs had a significant positive impact on activity level, fitness measures, HRQoL, and self-reported symptoms. E-DATs produced no significant improvements in inflammatory markers (ie, IL-6, TNF- $\alpha$, CRP, c-peptide) or cholesterol levels in 3 RCTs. ${ }^{37,39,44}$

\section{E-DATs, Self-Efficacy, and Adherence to \\ Exercise Interventions}

In recent years, DAT technology has advanced tremendously; $>1,200$ mobile health tools or apps have been developed, ${ }^{50}$ and wearable technology sales, including DATs, have increased from $<120$ million sales in 2013 to $>200$ million sales projected in 2018. ${ }^{51}$ DATs have the potential to improve healthcare delivery and education, facilitate data collection with real-time feedback, monitor patients remotely, ${ }^{52}$ and enable patients to self-monitor their exercise duration and intensity. Although exercise is known to ameliorate cancer- and treatment-related toxicities, incorporation of exercise interventions into routine clinical care for cancer survivors has been slow. ${ }^{53}$ Multiple studies ${ }^{44,54-56}$ have reported that patients prefer home-based, low-cost exercise interventions; however, though home-based unsupervised exercise may be more convenient $^{38}$ and cost-efficient, ${ }^{38,57}$ adherence may be lower than for supervised exercise programs.

Self-efficacy is a strong predictor of exercise adherence $^{15,18}$ and therefore an important goal for achieving the optimal benefits of exercise. ${ }^{13-15}$ Use of E-DATs enables healthcare providers to remotely monitor exercise sessions conducted at home and to provide real-time feedback. Consequently, incorporating DAT technology into a home-based exercise program can improve self-efficacy of cancer survivors, leading to higher adherence..$^{58}$ A recent systematic review in the general geriatric population showed that adherence to technology-based exercise programs is higher (91.25\%; range, $70.25 \%-100 \%)$ than for traditional exercise programs without E-DATs (83.58\%). ${ }^{59}$ This study showed adherence rates similar to those in our systematic review. Nevertheless, the varying adherence rates reported in our study highlight the need to consistently assess adherence to mode, frequency, amount, and intensity of exercise to strengthen the measures and comparisons between studies, ${ }^{60}$ and to better understand the cognitive-behavioral constructs that are mediators of adherence in this population.

\section{E-DATs, Activity Level, Physical Examination}

Findings, and Objective Fitness Outcomes

Our systematic review reported that E-DATs may improve activity levels and exercise capabilities of cancer survivors, including daily step counts, metabolic equivalent of task hours per week, and time spent engaged in brisk walking or moderate to vigorous activity, rather than sedentary behavior. We also found that E-DATs may reduce cardiovascular risk factors (eg, BMI and waist circumference) and optimize physical fitness (eg, $\mathrm{Vo}_{2 \max }$ and 6-minute walk test) among cancer survivors. These findings are noteworthy because cancer survivors often are at increased risk for short- and long-term sequelae of oncologic treatments, including obesity, cardiovascular disease, metabolic syndrome, osteopenia, and hypertension, all of which can be prevented or addressed with weight management and/or physical fitness. ${ }^{61,62}$ Numerous studies have shown that cancer survivors with better physical function were less likely to die prematurely than those with worse physical function. ${ }^{63,64}$

\section{E-DATs, HRQoL, and Self-Reported Symptoms}

Cancer survivors often develop treatment- and cancer-related symptoms, including fatigue, mental distress, and pain, which may negatively affect their HRQoL spanning social, physical, and mental function. ${ }^{65,66}$ We report that E-DATs significantly improve breast cancer-associated symptoms, ${ }^{39}$ fatigue, ${ }^{40,43}$ mood, ${ }^{43}$ vigor, ${ }^{43}$ well-being, ${ }^{44}$ and anxiety and depression. ${ }^{43,44}$ These findings are consistent with a recent meta-analysis ${ }^{8}$ showing that ex- 
ercise and psychological interventions are effective for reducing cancer-related fatigue during and after cancer treatment, and that these interventions are significantly better than the available pharmaceutical options. Additionally, several studies have reported that in patients with malignancy, physical activity level is directly associated with improved self-reported physiologic symptoms (pain, peripheral neuropathy, and fatigue) and psychological distress, anxiety, and depression, all of which contribute to HRQoL. ${ }^{6,58,67} \mathrm{HRQoL}$ is also consistently valued as a high priority by patients, in some instances equal in importance and priority to treatment efficacy, ${ }^{68,69}$ and HRQoL correlates with patient satisfaction scores. ${ }^{68,70}$

\section{E-DATs and Biomarker Data}

Our systematic review showed no significant impact of E-DATs on serum biomarkers, including IL-6, TNF- $\alpha$, CRP, c-peptide, and cholesterol profile in 3 RCTs, contrary to other studies that have reported increased IL-10, lower TNF- $\alpha$, and lower CRP levels in healthy populations. ${ }^{71-73}$ Our negative findings may be due to the chronically high inflammatory state driven by the presence of cancer and active chemotherapy.

\section{Ongoing E-DAT Studies in Cancer Survivors}

Supplemental eTable 5 summarizes numerous ongoing technology-based exercise interventions (available at ClinicalTrials.gov as of December 11, 2017). These studies focus primarily on solid malignancies, with few studies in hematologic malignancies. Most of these studies include a DAT in combination with a smartphone-based app or online platform to promote exercise adherence and self-efficacy.

\section{Strengths and Limitations}

A major strength of our review is that only RCTs were included. To minimize selection bias, 2 authors independently reviewed studies for inclusion and outcomes data. Our study, however, has several limitations. First, the English language restriction and terminology in our prespecified search may have excluded relevant publications. Second, the RCTs included in the systematic review were heterogeneous in regards to population of cancer survivors, type and duration of E-DAT, and measured outcomes, limiting our ability to perform a meta-analysis and generalize our findings to any one clinical setting. Future studies will need to address differences in preferences and barriers within the diverse population of adults with cancer, including those related to race, socioeconomic status, and geographic location. DAT technologies $^{74}$ vary in accuracy, and the underlying differences in activity tracker quality could contribute to the heterogeneity in adherence and other outcomes in our study. Third, the limited length of follow-up in the RCTs precludes our ability to determine the long-term impacts of E-DATs on the measured outcomes. Finally, most RCTs (75\%) in this systematic review compared E-DATs with a control group with usual care or nonexercise interventions. Thus, we cannot conclude that E-DATs improves exercise adherence compared with conventional exercise interventions without E-DATs. Confirmation of the benefits of E-DATs specifically in improving exercise adherence will require prospective studies comparing 3 arms: E-DATs, exercise interventions without E-DATs, and usual care controls. Furthermore, we can use the Sequential, Multiple Assignment, Randomized Trial (SMART) and Multiphase Optimization Strategy (MOST) study designs to evaluate for interactions between different components of a single proposed intervention. ${ }^{25}$

\section{Conclusions}

Our study shows that E-DATs are feasible to implement in cancer survivors, with the potential to positively impact numerous clinically meaningful outcomes. The increasing population of cancer survivors necessitates nonpharmacologic interventions, such as E-DATs, to reduce treatmentand cancer-related toxicities. Before their widespread implementation, studies should evaluate cost-effectiveness and determine the ideal E-DAT prescriptions for ameliorating short- and long-term symptoms experienced by cancer survivors. Although the development of E-DATs entails design and implementation costs, ${ }^{75,76}$ there may be significant positive financial implications for healthcare systems if E-DAT use leads to reduced side effects or toxicities in cancer survivors.

\section{Acknowledgments}

The authors would like to acknowledge Daniel Castillo for his assistance in performing the literature search and Susan Rosenthal for her editorial assistance.

\footnotetext{
Submitted March 28, 2018; accepted for publication September 7, 2018. Disclosures: The authors have disclosed that they have not received any financial considerations from any person or organization to support the preparation, analysis, results, or discussion of this article.

Funding: This work was supported by the $\mathrm{NCl}$, including funds from NCORP (UG1CA189961), NCORP supplement (U10CA037420), and R25CA102618.

Previous Presentation: This research was presented as a poster at the 2018 ASCO Cancer Survivorship Symposium; February 16-17, 2018; Orlando, Florida.

Author contributions: Study concept and design: Schaffer, Fung. Data acquisition: Schaffer, Panneerselvam, Loh, Herrmann. Manuscript preparation: All authors. Critical revision: All authors.

Correspondence: Chunkit Fung, MD, Division of Hematology/Oncology, Department of Medicine, Wilmot Cancer Institute, University of Rochester Medical Center, 601 Elmwood Avenue, Box 704, Rochester, NY 14642. Email: Chunkit_Fung@urmc.rochester.edu
} 


\section{References}

1. Denlinger CS, Sanft T, Baker KS, et al. Survivorship, Version 2.2017, NCCN Clinical Practice Guidelines in Oncology. J Natl Compr Canc Netw 2017;15:1140-1163.

2. de Moor JS, Mariotto AB, Parry C, et al. Cancer survivors in the United States: prevalence across the survivorship trajectory and implications for care. Cancer Epidemiol Biomarkers Prev 2013;22:561-570.

3. Stein KD, Syrjala KL, Andrykowski MA. Physical and psychological longterm and late effects of cancer. Cancer 2008;112:2577-2592.

4. Loh KP, Pandya C, Zittel J, et al. Associations of sleep disturbance with physical function and cognition in older adults with cancer. Support Care Cancer 2017;25:3161-3169.

5. Kleckner IR, Dunne RF, Asare M, et al. Exercise for toxicity management in cancer-a narrative review. Oncol Hematol Rev 2018;14:28-37.

6. Knols R, Aaronson NK, Uebelhart D, et al. Physical exercise in cancer patients during and after medical treatment: a systematic review of randomized and controlled clinical trials. J Clin Oncol 2005;23:3830-3842.

7. Strasser B, Steindorf K, Wiskemann J, et al. Impact of resistance training in cancer survivors: a meta-analysis. Med Sci Sports Exerc 2013:45:2080-2090.

8. Mustian KM, Alfano CM, Heckler C, et al. Comparison of pharmaceutical, psychological, and exercise treatments for cancer-related fatigue: a meta-analysis. JAMA Oncol 2017;3:961-968.

9. Stout NL, Baima J, Swisher AK, et al. A systematic review of exercise systematic reviews in the cancer literature (2005-2017). PM R 2017;9:S347-384

10. Kim TH, Chang JS, Kong ID. Effects of exercise training on physical fitness and biomarker levels in breast cancer survivors. J Lifestyle Med 2017;7:55-62.

11. Sweegers MG, Altenburg TM, Chinapaw MJ, et al. Which exercise prescriptions improve quality of life and physical function in patients with cancer during and following treatment? A systematic review and meta-analysis of randomised controlled trials. Br J Sports Med 2018;52:505-513

12. Kushi LH, Doyle C, McCullough M, et al. American Cancer Society Guidelines on nutrition and physical activity for cancer prevention: reducing the risk of cancer with healthy food choices and physical activity. CA Cancer J Clin 2012;62:30-67.

13. Kampshoff CS, Jansen F, van Mechelen W, et al. Determinants of exercise adherence and maintenance among cancer survivors: a systematic review. Int J Behav Nutr Phys Act 2014;11:80

14. Kampshoff CS, van Mechelen W, Schep G, et al. Participation in and adherence to physical exercise after completion of primary cancer treatment. Int J Behav Nutr Phys Act 2016;13:100.

15. Husebo AM, Karlsen B, Allan H, et al. Factors perceived to influence exercise adherence in women with breast cancer participating in an exercise programme during adjuvant chemotherapy: a focus group study. J Clin Nurs 2015;24:500-510.

16. Daley AJ, Crank H, Mutrie N, et al. Determinants of adherence to exercise in women treated for breast cancer. Eur J Oncol Nurs 2007;11:392-399

17. McAuley E, Mullen SP, Szabo AN, et al. Self-regulatory processes and exercise adherence in older adults: executive function and self-efficacy effects. Am J Prev Med 2011;41:284-290.

18. Neupert SD, Lachman ME, Whitbourne SB. Exercise self-efficacy and control beliefs: effects on exercise behavior after an exercise intervention for older adults. J Aging Phys Act 2009;17:1-16.

19. Larson JL, Covey MK, Kapella MC, et al. Self-efficacy enhancing intervention increases light physical activity in people with chronic obstructive pulmonary disease. Int J Chron Obstruct Pulmon Dis 2014;9:1081-1090.

20. Edmunds J, Ntoumanis N, Duda JL. Adherence and well-being in overweight and obese patients referred to an exercise on prescription scheme: a self-determination theory perspective. Psychol Sport Exerc 2007;8:722-740.

21. Rajati F, Sadeghi M, Feizi A, et al. Self-efficacy strategies to improve exercise in patients with heart failure: a systematic review. ARYA Atheroscler 2014;10:319-333.

22. Shaughnessy M, Resnick BM, Macko RF. Testing a model of post-stroke exercise behavior. Rehabil Nurs 2006;31:15-21.

23. Alharbi M, Gallagher R, Neubeck L, et al. Exercise barriers and the relationship to self-efficacy for exercise over 12 months of a lifestyle-change program for people with heart disease and/or diabetes. Eur J Cardiovasc Nurs 2017;16:309-317.
24. Ormel HL, van der Schoot GG, Sluiter WJ, et al. Predictors of adherence to exercise interventions during and after cancer treatment: a systematic review. Psychooncology 2018;27:713-724.

25. Evidence Generation Guide for Apps and Wearables Developers: Study Designs Including Applied Examples. Final Report. Available at: http:// www.yhec.co.uk/yhec-content/uploads/2017/03/YHEC-Study-Designs-28.03.17.pdf. Accessed December 1, 2018.

26. Kuijpers W, Groen WG, Aaronson NK, van Harten WH. A systematic review of web-based interventions for patient empowerment and physical activity in chronic diseases: relevance for cancer survivors. J Med Internet Res 2013;15:e37.

27. Patel MS, Asch DA, Volpp KG. Wearable devices as facilitators, not drivers, of health behavior change. JAMA 2015;313:459-460.

28. Powell $A C$, Landman $A B$, Bates DW. In search of a few good apps. JAMA 2014;311:1851-1852.

29. Bort-Roig J, Gilson ND, Puig-Ribera A, et al. Measuring and influencing physical activity with smartphone technology: a systematic review. Sports Med 2014;44:671-686.

30. Bantum EO, Albright CL, White KK, et al. Surviving and thriving with cancer using a Web-based health behavior change intervention: randomized controlled trial. J Med Internet Res 2014;16:e54.

31. Haggerty AF, Huepenbecker S, Sarwer DB, et al. The use of novel technology-based weight loss interventions for obese women with endometrial hyperplasia and cancer. Gynecol Oncol 2016;140:239-244.

32. Marshall G. The Story of Fitbit: How a Wooden Box Became a $\$ 4$ Billion Company. Available at: https://www.wareable.com/fitbit/youre-fitbitand-you-know-it-how-a-wooden-box-became-a-dollar-4-billion-company. Accessed December 2, 2018.

33. LaPorte RE, Montoye HJ, Caspersen CJ. Assessment of physical activity in epidemiologic research: problems and prospects. Public Health Rep 1985;100:131-146.

34. Vallance JK, Courneya KS, Plotnikoff RC, et al. Randomized controlled trial of the effects of print materials and step pedometers on physical activity and quality of life in breast cancer survivors. J Clin Oncol 2007:25:2352-2359.

35. Vallance JK, Courneya KS, Plotnikoff RC, et al. Analyzing theoretica mechanisms of physical activity behavior change in breast cancer survivors: results from the activity promotion (ACTION) trial. Ann Behav Med 2008;35:150-158.

36. Irwin ML, Cadmus L, Alvarez-Reeves M, et al. Recruiting and retaining breast cancer survivors into a randomized controlled exercise trial: the Yale Exercise and Survivorship Study. Cancer 2008;112:2593-2606.

37. Jones SB, Thomas GA, Hesselsweet SD, et al. Effect of exercise on markers of inflammation in breast cancer survivors: the Yale exercise and survivorship study. Cancer Prev Res (Phila) 2013;6:109-118.

38. Haines TP, Sinnamon $P$, Wetzig NG, et al. Multimodal exercise improves quality of life of women being treated for breast cancer, but at what cost? Randomized trial with economic evaluation. Breast Cancer Res Treat 2010;124:163-175

39. Backman $\mathrm{M}$, Wengstrom $\mathrm{Y}$, Johansson $\mathrm{B}$, et al. A randomized pilot study with daily walking during adjuvant chemotherapy for patients with breast and colorectal cancer. Acta Oncol 2014;53:510-520.

40. Mayo NE, Moriello C, Scott SC, et al. Pedometer-facilitated walking intervention shows promising effectiveness for reducing cancer fatigue: a pilot randomized trial. Clin Rehabil 2014;28:1198-1209.

41. James EL, Stacey FG, Chapman K, et al. Impact of a nutrition and physical activity intervention (ENRICH: Exercise and Nutrition Routine Improving Cancer Health) on health behaviors of cancer survivors and carers: a pragmatic randomized controlled trial. BMC Cancer 2015;15:710.

42. Park JH, Lee J, Oh M, et al. The effect of oncologists' exercise recommendations on the level of exercise and quality of life in survivors of breast and colorectal cancer: a randomized controlled trial. Cancer 2015;121:2740-2748.

43. Gokal K, Wallis D, Ahmed S, et al. Effects of a self-managed homebased walking intervention on psychosocial health outcomes for breast cancer patients receiving chemotherapy: a randomised controlled trial. Support Care Cancer 2016;24:1139-1166.

44. Jarden M, Moller T, Christensen KB, et al. Multimodal intervention integrated into the clinical management of acute leukemia improves physical function and quality of life during consolidation chemotherapy: a randomized trial 'PACE-AL'. Haematologica 2016;101:e316-319.

45. Sajid S, Dale W, Mustian K, et al. Novel physical activity interventions for older patients with prostate cancer on hormone therapy: a pilot randomized study. J Geriatr Oncol 2016;7:71-80.

46. Schwenk M, Grewal GS, Holloway D, et al. Interactive sensor-based 
balance training in older cancer patients with chemotherapy-induced peripheral neuropathy: a randomized controlled trial. Gerontology 2016;62:553-563.

47. Uhm KE, Yoo JS, Chung SH, et al. Effects of exercise intervention in breast cancer patients: is mobile health (mHealth) with pedometer more effective than conventional program using brochure? Breast Cancer Res Treat 2017;161:443-452.

48. Hills S. Nintendo Readies for "Wii Fit" Launch. Available at: https:// www.reuters.com/article/us-nintendo-wiifit/nintendo-readies-for-wii-fitlaunch-idUSN2152363320080222. Accessed December 2, 2018.

49. Eapen ZJ, Turakhia MP, McConnell MV, et al. Defining a mobile health roadmap for cardiovascular health and disease. J Am Heart Assoc 2016;5:pii: e003119.

50. Roess A. The promise, growth, and reality of mobile health - another data-free zone. N Engl J Med 2017;377:2010-2011.

51. Walker SR, Roashan R. Wearable Technology: The Small Revolution is Making Big Waves. Available at: https://technology.ihs.com/api/binary/526640. Accessed December 2, 2018.

52. Mohammadzadeh N, Safdari R. Patient monitoring in mobile health: opportunities and challenges. Med Arch 2014;68:57-60.

53. Santa Mina D, Alibhai SM, Matthew AG, et al. Exercise in clinical cancer care: a call to action and program development description. Curr Oncol 2012;19:e136-144.

54. Yardley L, Bishop FL, Beyer N, et al. Older people's views of falls-prevention interventions in six European countries. Gerontologist 2006;46:650-660.

55. Brawley LR, Rejeski WJ, King AC. Promoting physical activity for older adults: the challenges for changing behavior. Am J Prev Med 2003;25:172-183.

56. Karvinen $\mathrm{KH}$, Courneya $\mathrm{KS}$, Venner $\mathrm{P}$, et al. Exercise programming and counseling preferences in bladder cancer survivors: a population-based study. J Cancer Surviv 2007;1:27-34.

57. Cobiac LJ, Vos T, Barendregt JJ. Cost-effectiveness of interventions to promote physical activity: a modelling study. PLoS Med 2009;6:e1000110.

58. Albrecht TA, Taylor AG. Physical activity in patients with advanced-stage cancer: a systematic review of the literature. Clin J Onco Nurs 2012;16:293-300.

59. Valenzuela T, Okubo Y, Woodbury A, et al. Adherence to technology-based exercise programs in older adults: a systematic review. $J$ Geriatr Phys Ther 2018;41:49-61.

60. Hawley-Hague H, Horne M, Skelton D, et al. Review of how we should define (and measure) adherence in studies examining older adults' participation in exercise classes. BMJ Open 2016;6:e011560.
61. Jones LW, Eves ND, Haykowsky M, et al. Exercise intolerance in cancer and the role of exercise therapy to reverse dysfunction. Lancet Oncol 2009; 10:598-605.

62. Jones LW, Liu $\mathrm{Q}$, Armstrong GT, et al. Exercise and risk of major cardiovascular events in adult survivors of childhood Hodgkin lymphoma: a report from the childhood cancer survivor study. J Clin Oncol 2014;32:3643-3650.

63. Brown JC, Harhay MO, Harhay MN. Physical function as a prognostic biomarker among cancer survivors. Br J Cancer 2015;112:194-198.

64. Li T, Wei S, Shi Y, et al. The dose-response effect of physical activity on cancer mortality: findings from 71 prospective cohort studies. $\mathrm{Br} \mathrm{J}$ Sports Med 2016;50:339-345.

65. Mishra SI, Scherer RW, Snyder C, et al. Exercise interventions on health-related quality of life for people with cancer during active treatment. Cochrane Database Syst Rev 2012:CD008465.

66. Cella DF, Tulsky DS. Quality of life in cancer: definition, purpose, and method of measurement. Cancer Invest 1993;11:327-336.

67. Ferrer RA, Huedo-Medina TB, Johnson BT, et al. Exercise interventions for cancer survivors: a meta-analysis of quality of life outcomes. Ann Behav Med 2011;41:32-47.

68. Hitz F, Ribi K, Li Q, et al. Predictors of satisfaction with treatment decision, decision-making preferences, and main treatment goals in patients with advanced cancer. Support Care Cancer 2013;21:3085-3093.

69. Meropol NJ, Egleston BL, Buzaglo JS, et al. Cancer patient preferences for quality and length of life. Cancer 2008;113:3459-3466.

70. Asadi-Lari M, Tamburini M, Gray D. Patients' needs, satisfaction, and health related quality of life: towards a comprehensive model. Health Qual Life Outcomes 2004;2:32.

71. Gleeson M, Bishop NC, Stensel DJ, et al. The anti-inflammatory effects of exercise: mechanisms and implications for the prevention and treatment of disease. Nat Rev Immunol 2011;11:607-615.

72. Plaisance EP, Grandjean PW. Physical activity and high-sensitivity C-reactive protein. Sports Med 2006;36:443-458.

73. Petersen AM, Pedersen BK. The anti-inflammatory effect of exercise. J Appl Physiol 2005;98:1154-1162

74. Case MA, Burwick HA, Volpp KG, et al. Accuracy of smartphone applications and wearable devices for tracking physical activity data. JAMA 2015;313:625-626.

75. Fortuin J, Salie F, Abdullahi LH, et al. The impact of mHealth interventions on health systems: a systematic review protocol. Syst Rev 2016;5:200

76. Iribarren SJ, Cato K, Falzon L, et al. What is the economic evidence for mHealth? A systematic review of economic evaluations of $\mathrm{mHealth}$ solutions. Plos One 2017:12:e0170581. 